\title{
Alteration of microRNA profiling in sphere-cultured ovarian carcinoma cells
}

\author{
HWA JUN CHA ${ }^{1,2}$, SUNG KWAN AN ${ }^{2}$, TAE JIN KIM ${ }^{3,4}$ and JAE HO LEE ${ }^{4}$ \\ ${ }^{1}$ Department of Beauty Care and Cosmetics, Osan University, Osan, Gyeonggi 18119; ${ }^{2}$ Molecular-Targeted Drug \\ Research Center, Konkuk University, Seoul 05029; ${ }^{3}$ Department of Obstetrics and Gynecology; \\ ${ }^{4}$ Laboratory of Molecular Oncology, Cheil General Hospital and Women's Healthcare Center, \\ Dankook University College of Medicine, Seoul 04619, Republic of Korea
}

Received November 10, 2016; Accepted September 28, 2017

DOI: $10.3892 / \mathrm{ol} .2018 .8818$

\begin{abstract}
Ovarian cancer is an aggressive and lethal cancer, which in part, can be attributed to complications in the effective detection of this disease during early stages of progression. Frequently, epithelial ovarian cancer is disseminated to the abdominal cavity and forms multicellular aggregates. This unique early metastatic event, and formation of the multicellular aggregate is implicated to provide a basis for understanding the underlying molecular mechanisms of metastasis in ovarian cancer. Therefore, a 3-dimensional (3D) sphere culture system was established in the present study to mimic the later stages of ovarian cancer. The aim of the present study was to investigate whether microRNAs (miRNAs), which have functions in metastasis and chemoresistance in various cancer models, are altered in ovarian cancer cells by 3-dimensional (3D) culture. A multicellular aggregate of SKOV3ip1 ovarian carcinoma cells was generated using a 3D sphere culture system. Cell viability analysis demonstrated that the sphere-cultured SKOV3ip1 cells exhibited chemoresistance compared with those in a conventional 2-dimensional (2D) monolayer cultured SKOV3ip1 system. Under the same experimental conditions, 71 upregulated miRNAs and 63 downregulated miRNAs were identified in the 3D sphere-cultured SKOV3ip1 cells. The predicted targets of the 3D sphere-culture specific miRNAs were further identified using PITA, microRNAorg and TargetScan. Compared with the target gene pool and Kyoto Encyclopedia of Genes and Genomes pathway, the present study provides evidence that the $3 \mathrm{D}$ sphere culture-specific miRNAs regulated sphere formation and chemoresistance in
\end{abstract}

Correspondence to: Dr Jae Ho Lee, Laboratory of Molecular Oncology, Cheil General Hospital and Women's Heathcare Center, Dankook University College of Medicine, 17 Seoae-ro 1-gil, Jung, Seoul 04619, Republic of Korea

E-mail: jaeho3909@hanmail.net

Key words: ovarian cancer, microRNA, chemoresistance, 3-dimensional sphere culture systems
3D sphere-cultured SKOV3ip1 cells. Overall, the results of the present study demonstrated that miRNA-mediated regulation is implicated to provoke features of SKOV3ip1 multicellular aggregation, including sphere formation and chemoresistance.

\section{Introduction}

Ovarian cancer is categorized according to histology as serous, mucinous, endometrioid, clear cell, undifferentiated or unclassified carcinoma, or as a malignant Brenner tumor (1). Due to the lack of specific symptoms and effective detection methods, the majority of cases of ovarian cancer are detected at advanced stages (2). Surgery and chemotherapy using paclitaxel and platinum are representative treatments of ovarian cancer. In the early stages of disease, the aforementioned treatment rehabilitates $80-90 \%$ of patients $(2,3)$, and $10-15 \%$ of patients recover completely. However, $>70 \%$ of patients relapse and succumb within 5-years (2). In the later stages of disease, ovarian cancer spreads and attaches to the abdominal cavity, which is a common metastasis site for progressive ovarian cancer $(4,5)$. At the site of metastasis, ovarian cancer metastasizes to ascites as single cells or multicellular aggregates, which form spheroids $(6,7)$. The spheroids also exhibit chemoresistance to paclitaxel and platinum, which is implicated as a major contributory factor in relapse $(8,9)$. Thus, molecular studies using ovarian spheroids provide a robust model to elucidate the underlying mechanisms of chemoresistance and relapse following treatment in ovarian cancer. In previous studies, these spheroids have been mimicked by organotypic culture, 3-dimensional (3D) sphere culture systems, for understanding the mechanisms of spheroid development (10-12).

MicroRNAs (miRNAs/miRs) are small non-coding RNAs of 19-25 nucleotides in length that modulate gene silencing at the post-transcriptional level by interfering with translation and accelerating the degradation of specific target transcripts (13). These miRNAs are synthesized as pri-miRNA, which is sequentially cleaved to mature miRNA by RNase III enzymes, such as Drosha and Dicer. The mature miRNAs assemble into the RNA-induced silencing complex (RISC) (14). The RISC-mature miRNA complex is bound with the 3'-untranslated region (UTR) of specific target transcripts at the seed regions, which are located in the 5'-teminal of 
miRNA and complementarily matched with 3'UTR of target genes (15). Thus, the in silico miRNA target is predicted by the sequence of the seed site $(16,17)$. The miRNA-mediated translational interference is implicated in various physiological phenomena $(18,19)$. Notably, deregulation of miRNA expression is a major factor in the initiation, progression, metastasis and chemoresistance of a wide spectrum of different types of cancer $(20,21)$. For example, the let-7 family, commonly referred to as tumor suppressors, is downregulated in head, neck, lung, breast, ovarian and prostate cancer (22). Notably, in chemoresistant cancer, the miRNA expression pattern is definitively altered $(20,22)$. Thus, miRNAs are implicated as signature genetic biomarkers in chemoresistance of various types of cancer. In the present study, the alteration of miRNA expression in 3D sphere-cultured SK-OV3ip1 cells was examined, in addition to the investigating the association between the miRNA expression profile and characteristic features of 3D sphere-cultured SK-OV3ip1 cells.

\section{Materials and methods}

Cell culture. SKOV3ip1 cells were obtained from Professor A.K. Sood, University of Texas MD Anderson Cancer Center, (Texas, USA). Cells were cultured in RPMI 1640 medium (Corning Incorporated, Corning, NY, USA) supplemented with $10 \%$ fetal bovine serum (FBS, Invitrogen; Thermo Fisher Scientific, Inc., Waltham, MA, USA), $100 \mathrm{IU} / \mathrm{ml}$ penicillin and $100 \mu \mathrm{g} / \mathrm{ml}$ streptomycin (23). Cultures were incubated at $37^{\circ} \mathrm{C}$ in a humidified atmosphere of $5 \% \mathrm{CO}_{2}$. In addition, 3D sphere-cultured SKOV3ip1 cells were cultured in ultra-low attachment 6-well plates (Corning Incorporated), in the same culture conditions.

Cell viability assay. Cell viability was determined by crystal violet assay. To determine cell viability in conventional 2-dimensional (2D) monolayer cultures and a 3D sphere culture model, SKOV3ip1 cells were seeded at a density of $5 \times 10^{4}$ cells/well in conventional $24-w e l l$ plates and incubated with the indicated concentration $(0,6.25,12.5,25,50$ and $100 \mathrm{nM})$ of paclitaxel at $37^{\circ} \mathrm{C}$. At $72 \mathrm{~h}$ post-paclitaxel treatment, 3D sphere cultured-SKOV3ip1 cells were transferred to conventional 24-well plates and incubated for $12 \mathrm{~h}$ at $37^{\circ} \mathrm{C}$. Attached viable SKOV3ip1 cells were stained with $0.2 \%$ crystal violet solution for $5 \mathrm{~min}$ at $37^{\circ} \mathrm{C}$. For colorimetric analysis, crystal violet dye was extracted using $1 \%$ SDS/PBS and the absorbance was determined at $570 \mathrm{~nm}$ using an EMax PLUS microplate reader (Molecular Devices, LLC, Sunnyvale, CA, USA).

miRNA microarray. Total RNA was extracted from each sample (2D monolayer cultures and a 3D sphere cultured SKOV3ip1) using TRIzol reagent (Invitrogen; Thermo Fisher Scientific, Inc.) according to the manufacturer's protocol. Total RNAs were labeled with Cy3 using pCp (Agilent Technologies, Inc., Santa Clara, CA, USA) and an Agilent miRNA labeling kit (Agilent Technologies, Inc.). Salt and debris were removed using Micro Bio-Spin P-6 columns (Bio-Rad Laboratories, Inc., Hercules, CA, USA). Purified labeled total RNAs were loaded into SurePrint G3 Human V16 miRNA 8x60 K array (Agilent Technologies, Inc.), with 1,205 annotated miRNA sequences, and hybridized at $65^{\circ} \mathrm{C}$ for $20 \mathrm{~h}$. After hybridization, the microarray was washed with washing solution (Gene Expression Wash Buffer pack; Agilent Technologies, Inc.) and dried. The microarray was scanned using Agilent Microarray Scanner (Agilent Technologies, Inc.), and digitalized by Agilent Feature Extraction Software (Agilent Technologies, Inc.). Gene expression fold-change was analyzed by comparing between digitalized miRNA profile data using GeneSpring GX version 11.5 (Agilent Technologies, Inc.).

In silico analysis of putative target genes. Target genes of 3D sphere culture-specific miRNAs were identified by three online bioinformatics database, namely, PITA (http://genie. weizmann.ac.il), microRNAorg (http://www.microrna.org) and TargetScan (http://www.targetscan.org).

Kyoto Encyclopedia of Genes and Genomes (KEGG) analysis of putative targets. KEGG analysis of putative target genes was determined using the Database for Annotation, Visualization and Integrated Discovery Bioinformatics Resource 6.7 (DAVID, http://david.abcc.ncifcrf.gov). A list of putative target genes was uploaded and analyzed in DAVID for identifying associated KEGG pathways. Associated KEGG pathways were selected according to the threshold of the Expression Analysis Systematic Explorer Score $(<0.1)$, a modified Fisher's Exact P-Value.

Statistical analysis. The experimental data were statistically analyzed using the Student's t-test for two groups using Excel 2016 version 1,707 Microsoft Corporation, Redmond, WA, USA). $\mathrm{P}<0.05$ was considered to indicate a statistically significant difference. All experimental data are expressed as the mean \pm standard deviation from three independent experiments.

\section{Results}

Increased chemoresistance in spheroid culture model of SKOV3ipl cells. To compare miRNA expression profiles between conventional 2D monolayer-cultured SKOV3ip1 and 3D sphere-cultured SKOV3ip1, SKOV3ip1 cells were cultured in ultra-low attachment culture plates. As presented in Fig. 1, in the conventional 2D sphere culture system (Fig. 1A), SKOV3ip1 formed loose sheet-like aggregates and did not accumulate as compact spheroids, unlike in 3D monolayer-cultured SKOV3ip1 (Fig. 1B). To determine whether paclitaxel differentially affected the cell viability in 2D monolayer and 3D sphere culture, paclitaxel-induced cell cytotoxicity was evaluated in the two systems. The cell viability of conventional 2D monolayer-cultured and 3D sphere-cultured SKOV3ip1 cells was decreased by paclitaxel in a dose-dependent manner. However, colorimetric cell viability analysis demonstrated that 3D sphere-cultured SKOV3ip1 cells exhibited greater resistance to paclitaxel than monolayer-cultured SKOV3ip1 (Fig. 2).

Differences in miRNA expression profile between normal culture and spheroid culture. The miRNA expression profile was examined to compare differentially expressed miRNAs, between the conventional 2D monolayer-cultured SKOV3ip1 
Table I. Number of 3-dimensional sphere culture-specific miRNA targets using three miRNA target prediction databases.

\begin{tabular}{lcc}
\hline Database & Target miRNAs, $\mathrm{n}$ & Overlapping miRNAs in all three databases, $\mathrm{n}$ \\
\hline Upregulated miRNAs & & 96 \\
microRNAorg & 709 & \\
TargetScan & 808 & 662 \\
PITA & 156 & \\
Downregulated miRNAs & & \\
microRNAorg & 2,695 & \\
TargetScan & 1,465 & \\
PITA & 969 &
\end{tabular}

miRNAs, microRNAs.
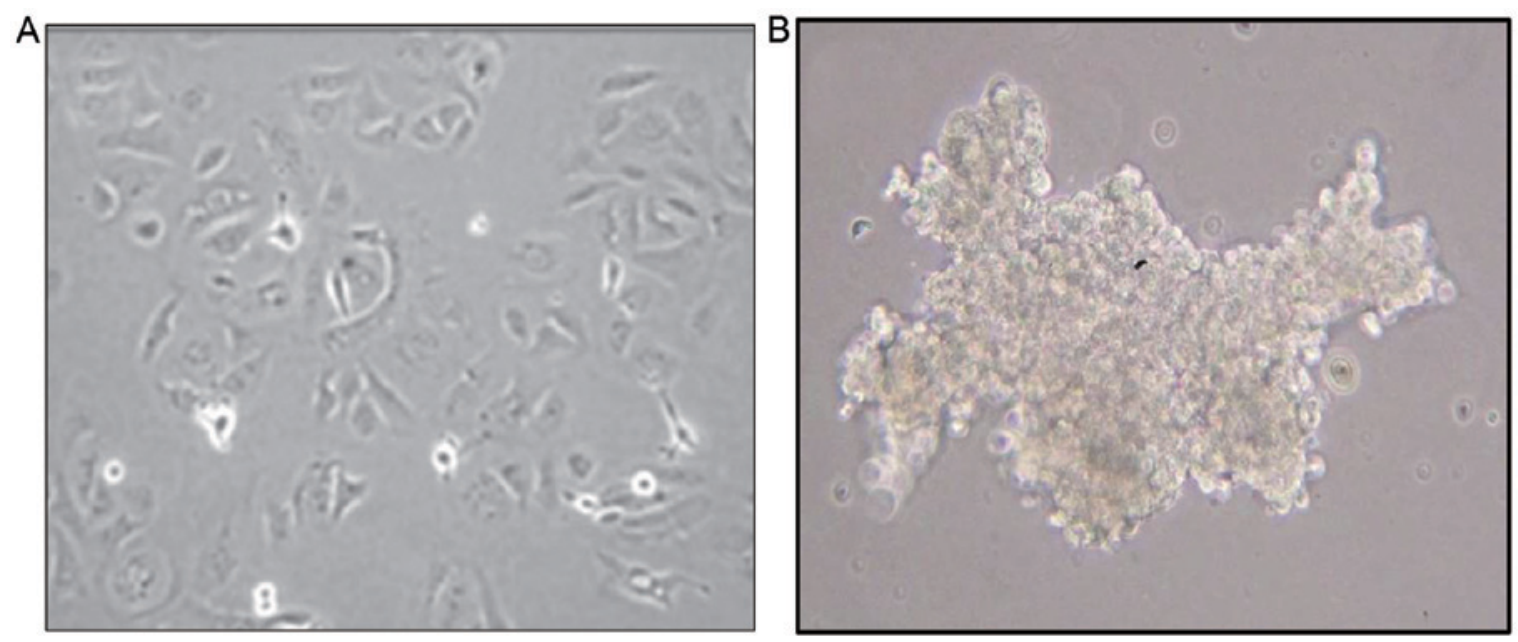

Figure 1. Morphology of SKOV3ip1 cultured by two-different culture systems. (A) Conventional 2-dimensional monolayer-cultured SKOV3ip1 cells. (B) 3-dimensional sphere-cultured SKOV3ip1 cells. Cell images were captured with a phase-contrast microscope at x100 magnification.

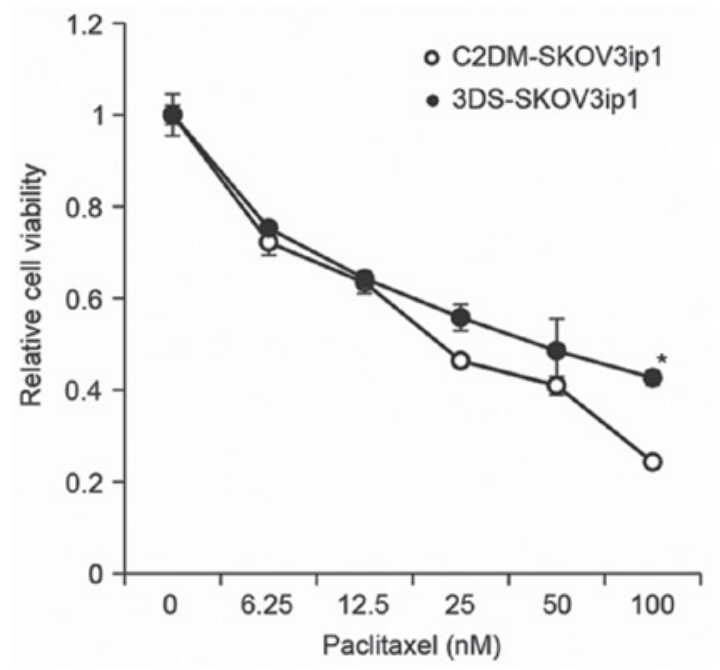

Figure 2. Effect of paclitaxel on cell viability in C2DM- and 3DS-cultured SKOV3ip1 cells. SKOV3ip1 cells were treated with paclitaxel at the indicated concentrations for $72 \mathrm{~h}$ in the conventional C2DM and 3DS. The results are representative of three independent experiments. ${ }^{*} \mathrm{P}<0.05$, compared with C2DM-SKOV3ip1. C2DM, conventional 2-dimensional monolayer-cultured system; 3DS, 3-dimensional sphere-cultured system. and 3D sphere-cultured SKOV3ip1 systems by SurePrint G3 Human V16 miRNA 8x60 K array, probed with 1,205 annotated miRNAs. As demonstrated in Fig. 3, 71 and 63 miRNAs were upregulated and downregulated, respectively, in the $3 \mathrm{D}$ spheroid culture model compared with the conventional 2D monolayer culture system in the SKOV3ip1 cells (Fig. 1). From 134 spheroid culture differentially expressed miRNAs, miR-3937 was the most upregulated by 965.61 fold, and miR-18a-5p was the most downregulated by 849.08 fold.

In addition, identified target genes of spheroid culturespecific miRNAs were identified using miRNA target gene prediction databases, namely, PITA, microRNAorg and TargetScan (Table I). A total of 96 putative target genes of spheroid culture-specific upregulated miRNAs were identified by all databases. Additionally, 662 putative target genes of spheroid culture-specific downregulated miRNAs were identified using all 3 target prediction systems. In addition, the analysis of the association between target genes of spheroid culture specific miRNAs and the biological functions of these target genes was conducted using KEGG pathways analysis. The data presented in Fig. 4 and Table II demonstrates that the target genes of specific upegulated 

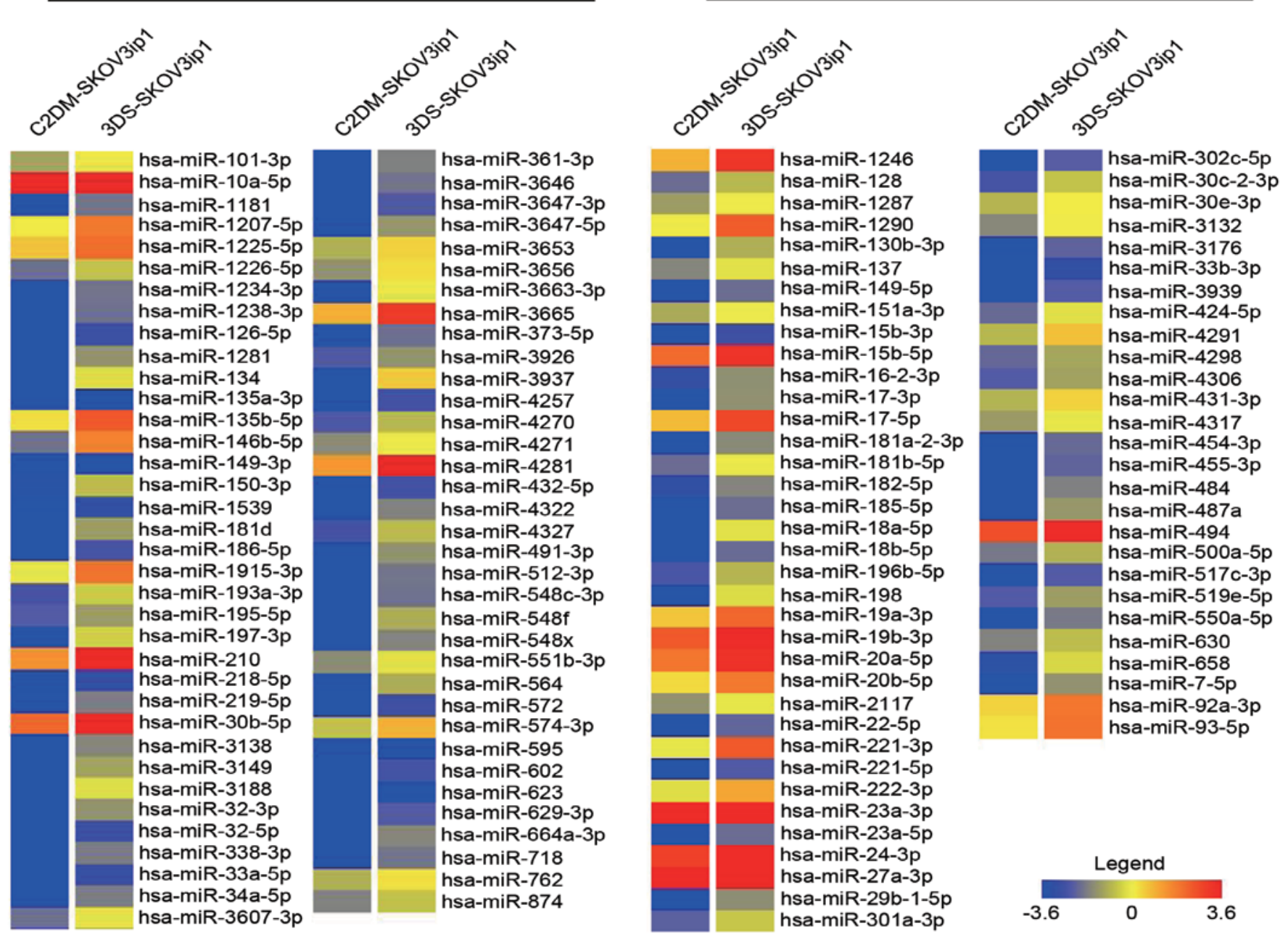

Figure 3. Alteration of miRNA profiling in conventional 2D monolayer- and 3D sphere-cultured SKOV3ip1. Heatmap exhibiting 134 miRNAs that were selected by filtering the microarray data. Expression levels are represented using red and blue, which represent high and low levels, respectively. C2DM, conventional 2-dimensional monolayer-cultured system; 3DS, 3-dimensional sphere-cultured system. miRNA/miR, microRNA.

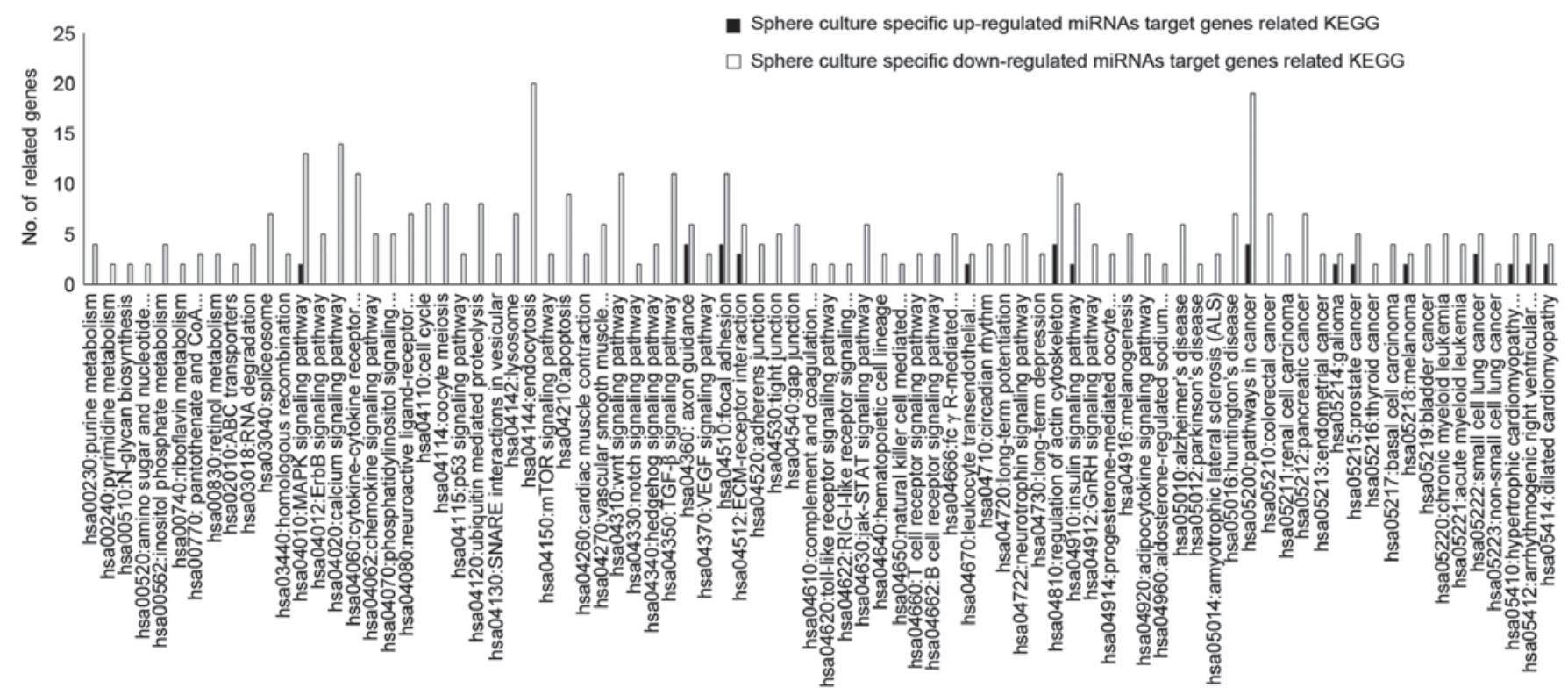

Figure 4. KEGG pathway analysis of sphere-specific miRNAs target genes. KEGG pathways are selected with target genes of upregulated and downregulated miRNAs using a calculated association score between KEGG pathway-contained genes and sphere-specific miRNA target genes using Database for Annotation, Visualization and Integrated Discovery Bioinformatics Resource. KEGG, Kyoto Encyclopedia of Genes and Genomes; miRNA, microRNA. 


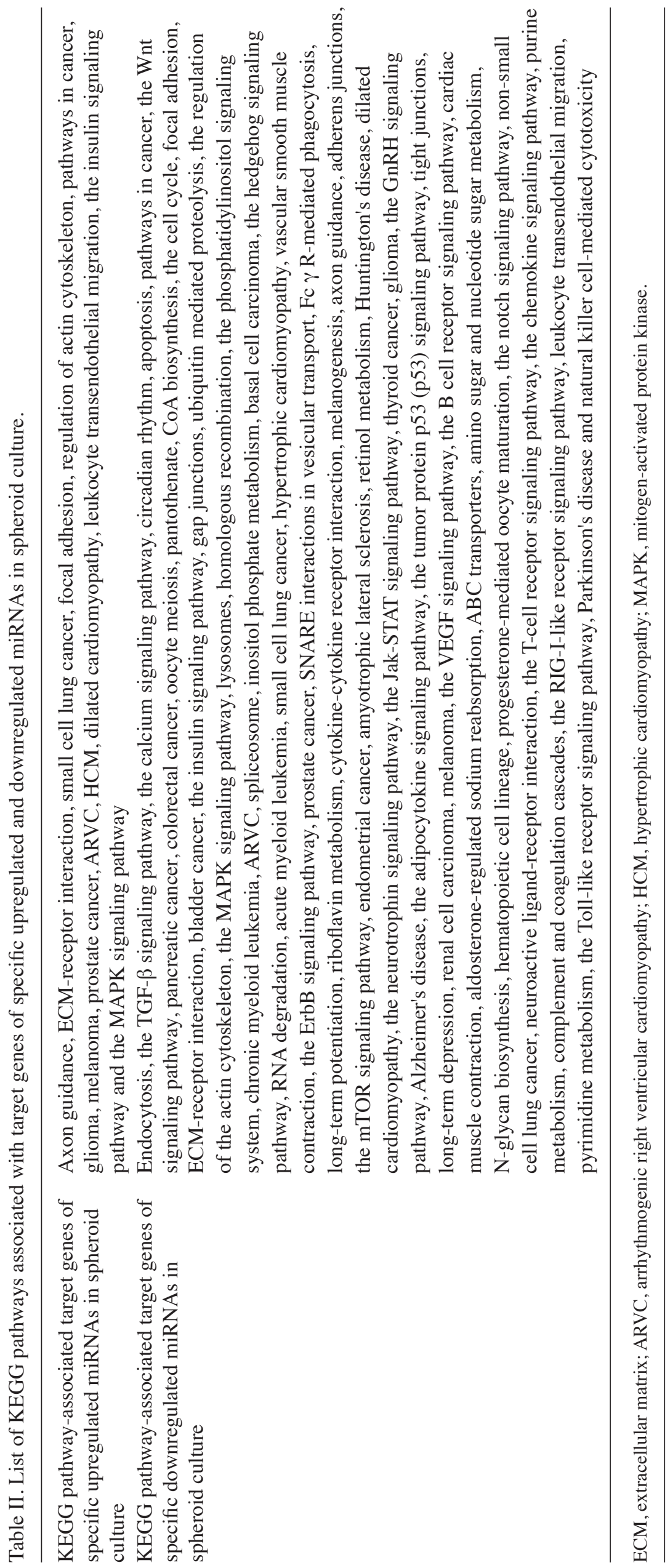


and downregulated miRNAs in spheroid culture were linked with various KEGG pathways.

\section{Discussion}

In gynecology, ovarian cancer is the most lethal malignancy $(1,2)$. Unlike for other types of cancer, the development of ovarian cancer is closely associated with a specific metastatic tumor microenvironment $(6,7)$. In an ovarian cancer-specific microenvironment, metastatic cell spheres gain a resistance to anticancer agents, including paclitaxel $(8,9)$. In particular, the emergence of chemoresistance in recurrent ovarian cancer is observed, which is a major hurdle in overcoming ovarian cancer $(8,9)$. Therefore, there is an urgent requirement to better understand the mechanisms underlying the formation of multicellular aggregation, and to overcome mechanisms of chemoresistance to anticancer drugs. In Figs. 1 and 2, an in vitro multicellular aggregation was generated using the 3D sphere culture system in SKOV3ipl cells. Additionally, it was demonstrated that 3D sphere-cultured SKOV3ip1 cells resisted paclitaxel-mediated cell death compared to 2D monolayer cultured SKOV3ip1 cells. In order to identify miRNAs that regulated the formation of multicellular aggregated 3D spheres and chemoresistance, the miRNA profile in conventional 2D monolayer-cultured SKOV3ip1 and 3D sphere-cultured SKOV3ip1 cells was displayed. As demonstrated in Fig. 3, of the 134 differentially expressed miRNAs identified, 71 were upregulated and 63 were downregulated in the $3 \mathrm{D}$ sphere culture system, compared with the conventional 2D monolayer culture system. To determine the association between miRNA-mediated regulation of target gene expression and 3D sphere cultivation of ovarian cancer, putative target genes of 3D sphere culture-specific miRNAs were identified (Fig. 3). Additionally, putative target genes of the 3D sphere culture-specific miRNA-associated KEGG pathway were analyzed (Fig. 4). Notably, the MAPK signaling pathway, regulation of the actin cytoskeleton, focal adhesion and pathways in cancer were highly associated with target genes of upregulated and downregulated miRNAs. The MAPK signaling pathway, regulation of the actin cytoskeleton and focal adhesion are implicated in anticancer drug chemoresistance (24-30). Paclitaxel induces p38 and ERK/MAPK-mediated apoptosis and cell death (25-27). Furthermore, p38, MAPK and epidermal growth factor receptor were constitutively activated in paclitaxel chemoresistance cells, which induces P38 MAPK-mediated E3 ubiquitin-protein ligase Mdm2 degradation and p53-mediated drug resistance (26). MAPK signaling-mediated anticancer drug chemoresistance is apparent in various types of cancer, including breast, ovarian and lung cancer $(25,26,28)$. Additionally, paclitaxel promotes microtubule assembly and stabilization, which is a key mechanism for inducing anticancer activity (29). Dysregulation of the interactions between microtubules and cell adhesion induced paclitaxel chemoresistance in ovarian cancer (30). Additionally, regulation of focal adhesion induced integrin-mediated spherical formation $(31,32)$. Thus, miRNA-mediated regulation of the MAPK signaling pathway, regulation of the actin cytoskeleton and focal adhesion induced paclitaxel resistance and sphere formation in 3D sphere-cultured SKOV3ip1 cells.
In conclusion, the results of the present study demonstrated that sphere-formed SKOV3ip1 cells acquired chemoresistance to paclitaxel and cell-cell interaction, which is associated with alteration of miRNA profiles in SKOV3ip1 ovarian cancer cells.

\section{Acknowledgements}

Not applicable.

\section{Funding}

The present study was supported by the Basic Science Research Program through the National Research Foundation of Korea funded by the Ministry of Education (grant no. 2015R1D1A1A01060688).

\section{Availability of data and materials}

The datasets used and/or analyzed during the current study are available from the corresponding author on reasonable request.

\section{Authors' contributions}

HJC, SKA, TJK and JHL conceived and designed the experiments. HJC performed the experiments. HJC, SKA, TJK and JHL performed the validation and formal analysis and curated and analyzed the data. HJC, SKA, TJK and JHL wrote the manuscript. HJC, SKA, TJK and JHL reviewed and revised the manuscript. JHL acquired funding. All authors have read and approved the final manuscript.

\section{Ethics approval and consent to participate}

Not applicable.

\section{Consent for publication}

Not applicable.

\section{Competing interests}

The authors declare that they have no competing interests.

\section{References}

1. Cannistra SA: Cancer of the ovary. N Engl J Med 351: 2519-2529, 2004.

2. Kindelberger DW, Lee Y, Miron A, Hirsch MS, Feltmate C, Medeiros F, Callahan MJ, Garner EO, Gordon RW, Birch C, et al: Intraepithelial carcinoma of the fimbria and pelvic serous carcinoma: Evidence for a causal relationship. Am J Surg Pathol 31: 161-169, 2007.

3. Yoshida S, Furukawa N, Haruta S, Tanase Y, Kanayama S, Noguchi T, Sakata M, Yamada Y, Oi H and Kobayashi H: Expression profiles of genes involved in poor prognosis of epithelial ovarian carcinoma: A review. Int J Gynecol Cancer 19: 992-997, 2009.

4. Saad AF, Hu W and Sood AK: Microenvironment and pathogenesis of epithelial ovarian cancer. Horm Cancer 1: 277-290, 2010.

5. Lengyel E: Ovarian cancer development and metastasis. Am J Pathol 177: 1053-1064, 2010.

6. Cormio G, Rossi C, Cazzolla A, Resta L, Loverro G, Greco P and Selvaggi L: Distant metastases in ovarian carcinoma. Int $\mathbf{J}$ Gynecol Cancer 13: 125-129, 2003. 
7. Naora $\mathrm{H}$ and Montell DJ: Ovarian cancer metastasis: Integrating insights from disparate model organisms. Nat Rev Cancer 5: 355-366, 2005

8. Weiswald LB, Bellet D and Dangles-Marie V: Spherical cancer models in tumor biology. Neoplasia 17: 1-15, 2015.

9. Yan XD, Li M, Yuan Y, Mao N and Pan LY: Biological comparison of ovarian cancer resistant cell lines to cisplatin and Taxol by two different administrations. Oncol Rep 17: 1163-1169, 2007.

10. Soriţău O, Tomuleasa CI, Páll E, Virág P, Fischer-Fodor E, Foris V, Barbos O, Tatomir C, Kacsó G and Irimie A: Enhanced chemoresistance and tumor sphere formation as a laboratory model for peritoneal micrometastasis in epithelial ovarian cancer. Rom J Morphol Embryol 51: 259-264, 2010.

11. Xu F, Celli J, Rizvi I, Moon S, Hasan T and Demirci U: A three-dimensional in vitro ovarian cancer coculture model using a high-throughput cell patterning platform. Biotechnol J 6: 204-212, 2011.

12. Xu G, Yin F, Wu H, Hu X, Zheng $\mathrm{L}$ and Zhao J: In vitro ovarian cancer model based on three-dimensional agarose hydrogel. J Tissue Eng 5: 2041731413520438, 2014. doi: $10.1177 / 2041731413520438$

13. Hannon GJ: RNA interference. Nature 418: 244-251, 2002.

14. Lehrbach NJ and Miska EA: Regulation of pre-miRNA Processing. Adv Exp Med Biol 700: 67-75, 2011.

15. Lenkala D, Gamazon ER, LaCroix B, Im HK and Huang RS: MicroRNA biogenesis and cellular proliferation. Transl Res 166 145-151, 2015.

16. Didiano D and Hobert O: Molecular architecture of a miRNA-regulated 3' UTR. RNA 14: 1297-1317, 2008.

17. Peterson SM, Thompson JA, Ufkin ML, Sathyanarayana $\mathrm{P}$, Liaw L and Congdon CB: Common features of microRNA target prediction tools. Front Genet 5: 23, 2014.

18. O'Connell RM, Rao DS, Chaudhuri AA and Baltimore D Physiological and pathological roles for microRNAs in the immune system. Nat Rev Immunol 10: 111-122, 2010.

19. Macfarlane LA and Murphy PR: MicroRNA: Biogenesis, function and role in cancer. Curr Genomics 11: 537-561, 2010.

20. Kutanzi KR, Yurchenko OV, Beland FA, Checkhun VF and Pogribny IP: MicroRNA-mediated drug resistance in breast cancer. Clin Epigenetics 2: 171-185, 2011.

21. Li SD, Zhang JR, Wang YQ and Wan XP: The role of microRNAs in ovarian cancer initiation and progression. J Cell Mol Med 14: 2240-2249, 2010.

22. Boyerinas B, Park SM, Hau A, Murmann AE and Peter ME: The role of let-7 in cell differentiation and cancer. Endocr Relat Cancer 17: F19-F36, 2010.
23. Yu D, Wolf JK, Scanlon M, Price JE and Hung MC: Enhanced c-erbB-2/neu expression in human ovarian cancer cells correlates with more severe malignancy that can be suppressed by E1A. Cancer Res 53: 891-898, 1993.

24. Zhao Y, Shen S, Guo J, Chen H, Greenblatt DY, Kleeff J, Liao Q, Chen G, Friess $\mathrm{H}$ and Leung PS: Mitogen-activated protein kinases and chemoresistance in pancreatic cancer cells. J Surg Res 136: 325-335, 2006.

25. Bacus SS, Gudkov AV, Lowe M, Lyass L, Yung Y, Komarov AP, Keyomarsi K, Yarden Y and Seger R: Taxol-induced apoptosis depends on MAP kinase pathways (ERK and p38) and is independent of p53. Oncogene 20: 147-155, 2001.

26. Park SH, Seong MA and Lee HY: p38 MAPK-induced MDM2 degradation confers paclitaxel resistance through p53-mediated regulation of EGFR in human lung cancer cells. Oncotarget 7 : 8184-8899, 2016

27. Okano J and Rustgi AK: Paclitaxel induces prolonged activation of the Ras/MEK/ERK pathway independently of activating the programmed cell death machinery. J Biol Chem 276: 19555-19564, 2001.

28. Ribeiro JR, Schorl C, Yano N, Romano N, Kim KK, Singh RK and Moore RG: HE4 promotes collateral resistance to cisplatin and paclitaxel in ovarian cancer cells. J Ovarian Res 9: 28, 2016.

29. McGrail DJ, Khambhati NN, Qi MX, Patel KS, Ravikumar N, Brandenburg CP and Dawson MR: Alterations in ovarian cancer cell adhesion drive taxol resistance by increasing microtubule dynamics in a FAK-dependent manner. Sci Rep 5: 9529, 2015.

30. Wang N, Zhang H, Yao Q, Wang Y, Dai S and Yang X: TGFBI promoter hypermethylation correlating with paclitaxel chemoresistance in ovarian cancer. J Exp Clin Cancer Res 31: 6, 2012.

31. Gallant ND, Michael KE and García AJ: Cell adhesion strengthening: Contributions of adhesive area, integrin binding, and focal adhesion assembly. Mol Biol Cell 16: 4329-4340, 2005.

32. Jin $\mathrm{H}$ and Varner J: Integrins: Roles in cancer development and as treatment targets. Br J Cancer 90: 561-565, 2004. 